\section{Surveillance of Central Venous Catheter- Associated Bloodstream Infection in a Scottish Hematology Unit}

To the Editor-In their recent article, Worth et al. ${ }^{1}$ suggest a new case definition for central venous catheter (CVC)-associated bloodstream infection (BSI) to be applied in hematology units. Their reasons for not using the established definitions from the National Nosocomial Infection Surveillance (NNIS) system ${ }^{2}$ are that they are complex and resource intensive. We wish to relate our experience in successfully establishing a CVC-associated BSI surveillance system in our unit using the NNIS definitions.

The hematology unit at Ninewells Hospital in Dundee, Scotland, has 13 beds and a day-patient area that cares for approximately 20 patients every weekday. During a 1-year period, data on CVC-associated BSI have been prospectively gathered. The unit's senior nurse has set up a database using Access (Microsoft) to record the number of patients with a Hickman (tunneled) catheter and the number of catheterdays per month. Information on the reason for any line being removed is also collected. The medical microbiologist (who is trained in infection control and who liaises with the unit regarding infections) performs a monthly inquiry for all positive blood culture results from the hematology unit, including the day-patient area, using the microbiology laboratory computer (LabCentre; CliniSys). Clinical information, entered into the computer by the medical microbiologists when they telephone in positive blood culture results, can be accessed to see details such as which antibiotic was used. The microbiologist then meets with the senior nurse monthly for approximately 1 hour in the hematology unit to review which patients with bacteremia meet the NNIS system diagnostic criteria for CVC-associated BSI. Any cases for which there is still doubt about the diagnosis, the medical notes can be referred to, as these are kept on the unit.

During the 1-year period (from April 2007 to March 2008), there were a total of a 29 cases of infection in 19 patients; 11 cases of infection fulfilled criterion 1 of the NNIS definitions, and 18 cases of infection fulfilled criterion 2. Coagulase-negative Staphylococcus was the sole organism found in the blood cultures for $17(58.6 \%)$ of the 29 cases of infection. The hematology unit has seen a decline in the incidence of CVC-associated BSI (albeit from a high level; see Figure), which may partly be due to the implementation of surveillance. The overall rate for that year was 12 cases of infection per 1,000 CVC-days.

A rate of 11.5 cases of infection per 1,000 CVC-days among hematology patients who used tunneled lines has been reported, ${ }^{3}$ although lower rates of infection have also been found. ${ }^{4}$ Despite possible contributing factors, such as neutropenia and whether the patient was hospitalized or not, ${ }^{5}$ we feel that the surveillance system we have implemented is worthwhile and has played a part in decreasing the incidence of CVC-associated BSI in our hematology unit. The key to

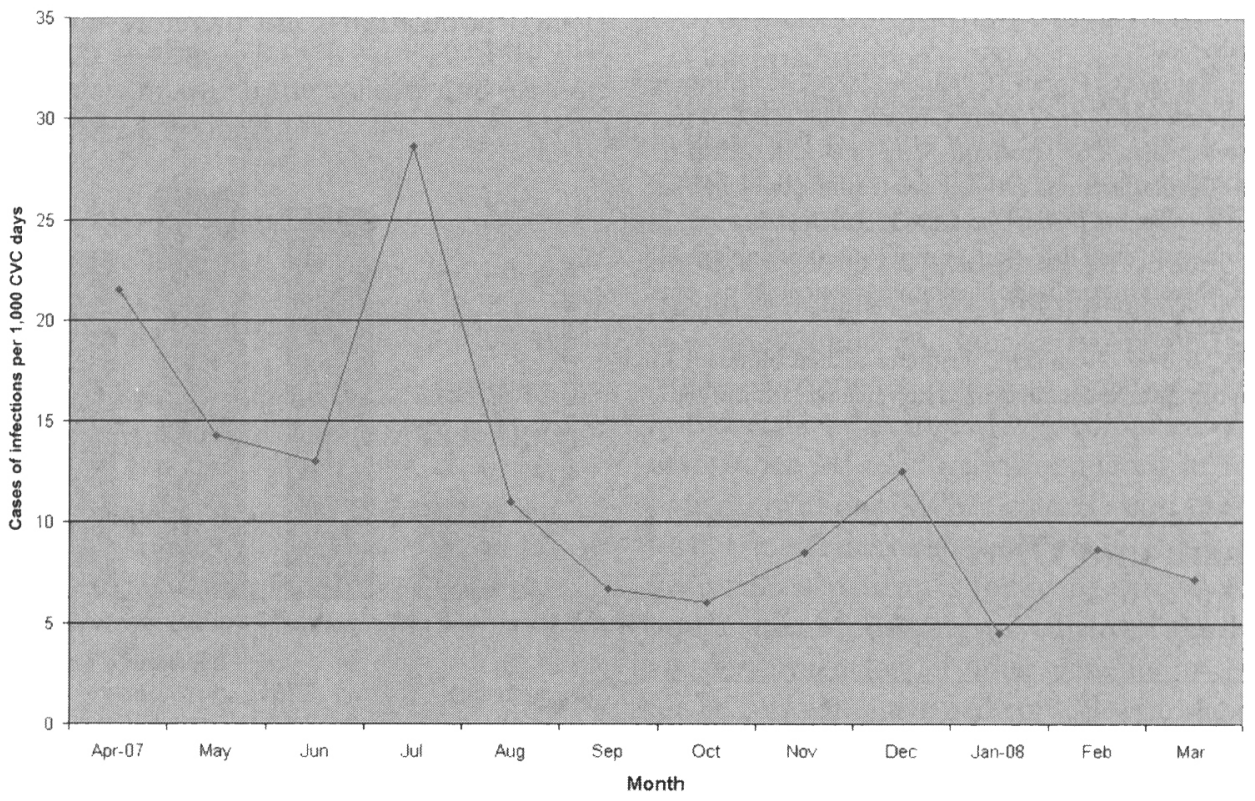
FIGURE. Monthly rates of central venous catheter (CVC)-associated bloodstream infection in the hematology unit at Ninewells Hospital
during the period from April 2007 to March 2008 . 
establishing successful surveillance has been the collaboration between the senior nurse, who was responsible for the unit's Hickman line protocols, and the medical microbiologist, who is familiar with NNIS definitions because of interaction with other hospital areas, such as the intensive care unit, and can ensure that the bacteremia data are complete. Use of the NNIS definitions has not been onerous, and has the advantage of being well established in many countries, and therefore data can be compared between centers. Creating separate case definitions for CVC-associated BSI in patients in a hematology unit could be counterproductive, because units considering using surveillance, faced with an expanding choice of definitions, may be less likely to do it at all.

\section{ACKNOWLEDGMENTS}

Potential conflicts of interest. All authors report no conflicts of interest relevant to this article.

Ann Graham, RN; William J. Olver, FRCPath

From the Department of Hematology (A.G.) and the Department of Medical Microbiology (W.J.O), Ninewells Hospital and Medical School, Dundee, United Kingdom.

Address reprint requests to William J Olver, FRCPath, Department of Medical Microbiology, Ninewells Hospital and Medical School, Dundee DD1 9SY, Scotland, UK (william.olver@nhs.net).

Infect Control Hosp Epidemiol 2008; 29:985-986

(C) 2008 by The Society for Healthcare Epidemiology of America. All rights reserved. 0899-823X/2008/2910-0019\$15.00.DOI: 10.1086/590391

\section{REFERENCES}

1. Worth LJ, Black J, Seymour JF, Thursky KA, Slavin MA. Surveillance for catheter-associated bloodstream infection in hematology units: quantifying the characteristics of a practical case definition. Infect Control Hosp Epidemiol 2008; 29:358-360.

2. Garner JS, Jarvis WR, Emori TG, Horan TC, Hughes JM. CDC definitions for nosocomial infections, 1988. Am J Infect Control 1988; 16:128-140.

3. Keung YK, Watkins K, Chen SC, Grosham S, Levine AM, Douer D. Increased incidence of central venous catheter-related infections in bone marrow transplant patients. Am J Clin Oncol 1995; 18:469-474.

4. Adler A, Yaniv I, Steinberg R, et al. Infectious complications of implantable ports and Hickman catheters in paediatric haematology-oncology patients. J Hosp Infect 2006; 62:358-365.

5. Worth LJ, Slavin MA, Brown GV, Black J. Catheter-related bloodstream infections in hematology: time for standardized surveillance? Cancer 2007; 109:1215-1226.

\section{Reply to Graham and Olver}

To the Editor-We thank Graham and Olver ${ }^{1}$ for their interest in our study ${ }^{2}$ of catheter-associated bloodstream infection (BSI) in hematology units, and for reporting their own experience of successfully applying the National Nosocomial
Infection Surveillance (NNIS) system definition in a Scottish hematology unit. We agree that a standard definition must be employed and that this is essential if benchmarking is to be performed, and we do not support the use of ad hoc or poorly validated case definitions.

Evidently, the work flow and size of the Scottish hematology unit enabled collaboration and regular review by a medical microbiologist. It is not clear, however, how many other hematology units have devoted nursing staff and a medical microbiologist with sufficient time to perform surveillance activities for infection. It would be helpful to know the number of hours required of these nurses during the surveillance period, as a measure of resource requirements. During the first 6 weeks of our study, the number of hours required for review by an infection control practitioner for application of NNIS methodology was monitored (see Figure), and the mean number of hours required was 1.6 hours per 10 beds per week.

Furthermore, Graham and Olver ${ }^{1}$ report experience with long-term central venous catheters (CVCs; ie, Hickman catheters), for which data on dates of insertion and removal may be more readily available to assist with the calculation of the denominator (ie, number of devices used per 1,000 CVCdays). In contrast, we studied medium-term $\mathrm{CVCs}^{2}$ (peripherally inserted and nontunneled), of which a larger number of individual devices are used, recording of the dates of insertion and removal may not be as reliable, and closer direct monitoring is required by surveillance staff to ensure accurate data collection. A standardized strategy must be practical for a range of tunneled, nontunneled, and implanted devices, if it is to be applied to a more broad population of hematology patients. ${ }^{3}$

NNIS methods have been employed in intensive care unit (ICU) populations, and therefore interhospital comparisons

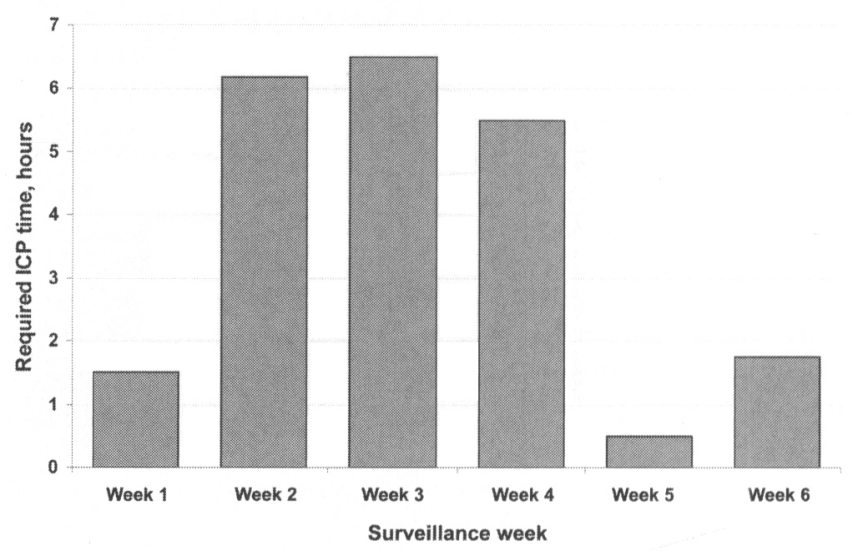

FIGURE. Number of hours required for surveillance of central venous catheter-associated bloodstream infection by an infection control practitioner (ICP) during the first 6 weeks of our study ${ }^{2}$ in a 23-bed hematology unit. 